\title{
Correction to: Rapid and simple colorimetric detection of multiple influenza viruses infecting humans using a reverse transcriptional loopmediated isothermal amplification (RT-LAMP) diagnostic platform
}

Su Jeong Ahn ${ }^{1 \dagger}$, Yun Hee Baek ${ }^{1 \dagger}$, Khristine Kaith S. Lloren', Won-Suk Choi ${ }^{1}$, Ju Hwan Jeong ${ }^{1}$, Khristine Joy C. Antigua', Hyeok-il Kwon', Su-Jin Park', Eun-Ha Kim', Young-il Kim', Young-Jae Si', Seung Bok Hong ${ }^{2}$, Kyeong Seob Shin ${ }^{3}$, Sungkun Chun ${ }^{4}$, Young Ki Choi ${ }^{1 *}$ and Min-Suk Song ${ }^{1 *}$

Correction to: BMC Infect Dis 19, 676 (2019)

https://doi.org/10.1186/s12879-019-4277-8

Following publication of the original article [1], the authors noticed that in Table 1, two primer sequences (BLF and H5-LF) should have been reverse complemented.

The sequences to correct are below marked:

The correct sequences should be indicated as below:

\begin{tabular}{cll}
\hline No. Needs Correction: & $\begin{array}{l}\text { Corrected } \\
\text { version }\end{array}$ \\
1. & Table 1/ B (NA & CTACAGGC \\
gene)/B-LF & GATGTCCGTGTAAGATACCAA & ACATTCTATG \\
Sequence: & GTT
\end{tabular}

Comment: Based on the reference in designing the forward loop (LF) primer: LF 5'-AACCATAGAATGTGCCTGTAG-3' (Figure 1, page 4), this identified sequence should have been reverse complemented however inadvertently the submitted sequence for the mentioned B-LF primer was only reversed.

The original article can be found online at https://doi.org/10.1186/s12879019-4277-8.

* Correspondence: choiki55@chungbuk.ac.kr; songminsuk@chungbuk.ac.kr ${ }^{\dagger}$ Su Jeong Ahn and Yun Hee Baek contributed equally to this work. 'Department of Microbiology, College of Medicine and Medical Research, Institute, Chungbuk National University, Chungdae-ro 1, Seowon-Ku, Cheongju 28644, Republic of Korea

Full list of author information is available at the end of the article

\begin{tabular}{|c|c|c|c|}
\hline 2. & $\begin{array}{l}\text { Table 1/ A/H5 (HA } \\
\text { gene)/ H5-LF } \\
\text { Sequence: }\end{array}$ & CTACCAACCATACCCATGG & $\begin{array}{l}\text { GGTACCCA } \\
\text { TACCAACCAT } \\
\text { C }\end{array}$ \\
\hline
\end{tabular}

Comment: Based on the reference in designing the forward loop (LF) primer: LF 5'-GATGGTTGGTATGGGTACC-3' (Figure 1, page 4), this identified sequence should have been reverse complemented however inadvertently the submitted sequence for the mentioned H5-LF primer was only reversed.

\section{Author details \\ ${ }^{1}$ Department of Microbiology, College of Medicine and Medical Research, Institute, Chungbuk National University, Chungdae-ro 1, Seowon-Ku, Cheongju 28644, Republic of Korea. ${ }^{2}$ Department of Clinical Laboratory, Science, Chungbuk Health and Science University, Cheongju, Republic of Korea. ${ }^{3}$ Departments of Laboratory Medicine, Chungbuk National University, College of Medicine, Cheongju, Republic of Korea. ${ }^{4}$ Department of Physiology, Chonbuk National University Medical School, Jeonju 54907, Republic of Korea.}

Published online: 24 December 2020

\section{Reference}

1. Ahn, et al. BMC Infect Dis. 2019;19:676. https://doi.org/10.1186/s12879-0194277-8.

(-) The Author(s). 2020 Open Access This article is licensed under a Creative Commons Attribution 4.0 International License, which permits use, sharing, adaptation, distribution and reproduction in any medium or format, as long as you give appropriate credit to the original author(s) and the source, provide a link to the Creative Commons licence, and indicate if changes were made. The images or other third party material in this article are included in the article's Creative Commons licence, unless indicated otherwise in a credit line to the material. If material is not included in the article's Creative Commons licence and your intended use is not permitted by statutory regulation or exceeds the permitted use, you will need to obtain permission directly from the copyright holder. To view a copy of this licence, visit http://creativecommons.org/licenses/by/4.0/ The Creative Commons Public Domain Dedication waiver (http://creativecommons.org/publicdomain/zero/1.0/) applies to the data made available in this article, unless otherwise stated in a credit line to the data. 
Table 1 Reverse transcriptional loop-mediated isothermal amplification (RT-LAMP) primers for detection of influenza subtypes

\begin{tabular}{|c|c|c|c|c|c|c|}
\hline Target & Gene & $\begin{array}{l}\text { Primer } \\
\text { name }\end{array}$ & Sequence(5'-3') & $\begin{array}{l}\text { Primer final } \\
\text { concentration }\end{array}$ & Gene position & $\begin{array}{l}\text { Length } \\
\text { (mer) }\end{array}$ \\
\hline \multirow[t]{6}{*}{$\mathrm{B}$} & NA & $\mathrm{B}-\mathrm{F} 3$ & CAGGAAGAGTAAAACATACTGAGGA & $5 \mu \mathrm{M}$ & $856-881$ & 25 \\
\hline & & B-B3 & GATTCGCAAGGCCCTGTT & $5 \mu \mathrm{M}$ & $1052-1069$ & 18 \\
\hline & & B-FIP & AGGGTCTITITGCTGTGTAACTGTT-GCACATGCGGATTTGCCAG & $40 \mu \mathrm{M}$ & $933-955+883-901$ & 44 \\
\hline & & B-BIP & GTGGAGACTGATACAGCGGAA-TGCTTCCATCATTTGGTCTGG & $40 \mu \mathrm{M}$ & $972-992+1030-1050$ & 42 \\
\hline & & B-LF & CTACAGGCACATTCTATGGTT & $10 \mu \mathrm{M}$ & $908-928$ & 21 \\
\hline & & $B-L B$ & ATAAGATTGATGTGCACA & $10 \mu \mathrm{M}$ & $993-1010$ & 18 \\
\hline \multirow[t]{6}{*}{$\mathrm{A} / \mathrm{H} 1$} & $\mathrm{HA}$ & $\mathrm{H} 1-\mathrm{F3}$ & AGCAAGAAGTTCAAGCCG & $5 \mu \mathrm{M}$ & $619-639$ & 18 \\
\hline & & H1-B3 & CGTGAACTGGTGTATCTGAA & $5 \mu \mathrm{M}$ & $801-820$ & 20 \\
\hline & & $\mathrm{H} 1-\mathrm{FIP}$ & GGCTCTACTAGTGTCCAGTAATAGT-AAATAGCAATAAGACCCAAAGTG & $80 \mu \mathrm{M}$ & $734-758+689-711$ & 48 \\
\hline & & H1-BIP & ATAACATTCGAAGCAACTGGAAATC-TGATAATACCAGATCCAGCATT & $80 \mu \mathrm{M}$ & $718-742+778-799$ & 47 \\
\hline & & H1-LF & TCTCCCTTCTTGATCCC & $10 \mu \mathrm{M}$ & $713-729$ & 17 \\
\hline & & $\mathrm{H} 1-\mathrm{LB}$ & TAGTGGTACCGAGATATGCA & $10 \mu \mathrm{M}$ & $794-813$ & 20 \\
\hline \multirow[t]{6}{*}{$\mathrm{A} / \mathrm{H} 3$} & $\mathrm{HA}$ & $\mathrm{H} 3-\mathrm{F} 3$ & GGGGTTACTTCAAAATACAAG & $5 \mu \mathrm{M}$ & $841-859$ & 19 \\
\hline & & H3-B3 & GTTGCCAATTTCAGAGTGTT & $5 \mu \mathrm{M}$ & $1011-1028$ & 18 \\
\hline & & H3-FIP & GAGTGATGCATTCAGAATTGCATTT-TGGGAAAAGCTCAATAATGAGA & $40 \mu \mathrm{M}$ & $903-927+863-884$ & 47 \\
\hline & & H3-BIP & AATGGAAGCATTCCCAATGACA-GCTTAACATATCTGGGACAGG & $40 \mu \mathrm{M}$ & $930-951+988-1008$ & 43 \\
\hline & & H3-LF & CCAATGGGTGCATCTGA & $10 \mu \mathrm{M}$ & $885-901$ & 17 \\
\hline & & H3-LB & AACCATTCCAAAATGTAAAC & $10 \mu \mathrm{M}$ & $952-971$ & 20 \\
\hline \multirow[t]{6}{*}{$\mathrm{A} / \mathrm{H} 5$} & $\mathrm{HA}$ & $\mathrm{H} 5-\mathrm{F} 3$ & GCTATAGCAGGTITTATAGAGG & $5 \mu \mathrm{M}$ & $1048-1069$ & 22 \\
\hline & & H5-B3 & GCCTCAAACTGAGTGTTCAT & $5 \mu \mathrm{M}$ & $1210-1229$ & 20 \\
\hline & & H5-FIP & ACTCCCCTGCTCATTGCTAT-GGATGGCAGGGAATGGTA & $80 \mu \mathrm{M}$ & $1112-1131+1072-1089$ & 38 \\
\hline & & H5-BIP & GGTACGCTGCAGACAAAGAAT-TGAGTTGACCTTATTGGTGAC & $80 \mu \mathrm{M}$ & $1133-1153+1177-1197$ & 42 \\
\hline & & H5-LF & GGTACCCATACCAACCATC & $5 \mu \mathrm{M}$ & $1093-1114$ & 19 \\
\hline & & H5-LB & CCACTCAAAAGGCAATAGATGGA & $5 \mu \mathrm{M}$ & $1154-1176$ & 23 \\
\hline \multirow[t]{6}{*}{$\mathrm{A} / \mathrm{H7}$} & $\mathrm{HA}$ & $\mathrm{H} 7-\mathrm{F3}$ & GCGGGTTTCATTGAAAATGG & $5 \mu \mathrm{M}$ & $1036-1055$ & 20 \\
\hline & & H7-B3 & СTACСTCATTGAATTCATTGTCT & $5 \mu \mathrm{M}$ & $1215-1237$ & 23 \\
\hline & & H7-FIP & TCCCTCTCCCTGTGCATTCT-ATGGGAAGGCCTAATTGATG & $80 \mu \mathrm{M}$ & $1097-1116+1056-1075$ & 40 \\
\hline & & H7-BIP & ACTGCTGCAGATTACAAAAGCAC-TGGTTGGTITITCTATAAGCC & $80 \mu \mathrm{M}$ & $1117-1139+1178-1199$ & 45 \\
\hline & & H7-LF & TCTGAAACCATACCAAC & $5 \mu \mathrm{M}$ & $1076-1092$ & 17 \\
\hline & & H7-LB & TCAATCGGCAATTGATCAAATA & $5 \mu \mathrm{M}$ & $1140-1161$ & 22 \\
\hline
\end{tabular}

